\title{
Transoral Videolaryngoscopic Surgery with a Navigation System for Excision of a Metastatic Retropharyngeal Lymph Node
}

\author{
Yuya Tanaka Masayuki Tomifuji Hiroshi Suzuki Taku Yamashita \\ Koji Araki Akihiro Shiotani \\ Department of Otolaryngology - Head and Neck Surgery, National Defense Medical \\ College, Tokorozawa, Japan
}

\section{Key Words}

Metastatic retropharyngeal lymph node - Squamous cell carcinoma of the tongue .

Transoral approach · Transoral videolaryngoscopic surgery · Navigation system

\begin{abstract}
Objectives: To report the utilization of a new transoral surgery system, i.e. transoral videolaryngoscopic surgery (TOVS) with a navigation system, for the removal of a metastatic retropharyngeal lymph node (RPLN). Case Report: An 86-year-old woman with tongue cancer (cT2NOM0) underwent partial glossectomy and left selective neck dissection. Three months postoperatively, a left metastatic RPLN was identified on follow-up magnetic resonance imaging. The metastatic RPLN was successfully excised by TOVS with a navigation system. After postoperative irradiation, she had no recurrence more than 1 year after the surgery. Discussion: The retropharyngeal space (RPS) is a difficult area to access surgically. A transcervical approach is conventionally used to access the RPS; however, in the present case, scar tissue after neck dissection may have been problematic. Visualization of the RPS by high-definition endoscopy and a navigation system were effective in facilitating the safe performance of the surgery. Conclusions: A transoral approach may be a viable choice for surgery of a metastatic RPLN. Surgical indications for a transoral approach to the RPS include that (1) the location of the RPLN is below the level of the hard palate and the pterygoid hamulus and (2) there is only a single lesion, without adhesion to the surrounding tissue.

(c) 2015 S. Karger AG, Basel
\end{abstract}


Tanaka et al.: Transoral Videolaryngoscopic Surgery with a Navigation System for Excision of a Metastatic Retropharyngeal Lymph Node

\section{Introduction}

It has been reported that metastases to the retropharyngeal lymph nodes (RPLNs) were associated with $20-50 \%$ of surgically treated oropharyngeal, hypopharyngeal, and cervical esophageal carcinomas [1-4]. A surgical approach to the retropharyngeal space (RPS), which is located in the deepest region of the head and neck, is generally difficult, so adequate tumor visualization and precise surgical orientation must be ensured for avoidance of damage to the surrounding critical nerves and vessels. A transcervical approach, as an extension of neck dissection, is conventionally chosen to access the RPS. Ozlugedik et al. [5] suggested that transcervical approaches should be divided into two groups: medial and lateral. In the medial approach, the branches of the external carotid artery may require ligation to achieve maximum surgical exposure. In the lateral approach, the surgical field is more limited than in the medial approach, although the branches of the external carotid artery can be preserved. In cases of reoperation after neck dissection, however, scar tissue is usually problematic with regard to reaching the RPS via a transcervical approach.

Since 2004, we have been developing a new transoral surgery system for laryngopharyngeal lesions [6-8]. In this system, a surgical assistant manages a rigid videolaryngoscope (Karl Storz Endoscopy, Germany) or a rigid endoscope with a flexible tip (ENDEYE FLEX, Olympus, Japan) to obtain the appropriate view. In operations involving the deep areas of the pharynx and larynx, the operator manipulates laparoscopic surgical instruments including the forceps, scissors, and electrocautery needles watching the endoscopic view on a monitor. This system allows the surgeon to obtain a wide surgical field and view. We have named this procedure transoral videolaryngoscopic surgery (TOVS). We herein report a case in which TOVS with a navigation system was very useful for the excision of a delayed metastatic RPLN in a patient with squamous cell carcinoma (SCC) of the tongue.

\section{Case Report}

An 86-year-old woman complained of pain in her tongue and visited the Department of Otolaryngology - Head and Neck Surgery at the National Defense Medical College in Tokorozawa, Japan. On physical examination, a tumor of approximately $2 \mathrm{~cm}$ in diameter was observed in the left margin of her tongue. Biopsy of the lesion showed SCC. The disease was staged as clinical T2N0M0 by contrast-enhanced computed tomography (CT), magnetic resonance imaging (MRI) and ${ }^{18} \mathrm{~F}$-fluorodeoxyglucose positron emission tomography/CT $\left({ }^{18} \mathrm{~F}\right.$-FDG PET/CT). ${ }^{99 \mathrm{~m}} \mathrm{Tc}$-radiocolloid lymphoscintigraphy was performed for sentinel node detection before partial glossectomy. Frozen section pathology of the biopsied sentinel node revealed that left level I and left level IIA were metastasis positive, so ipsilateral selective neck dissection including levels I-IV was added. The postoperative pathological stage was pT1pN2b. Although multiple lymph node metastases were detected, adjuvant radiation therapy was not performed, in accordance with the patient's wishes. Three months postoperatively, a follow-up MRI showed an enlarged left RPLN nearly $2.0 \mathrm{~cm}$ in diameter with well-defined borders (fig. 1a). ${ }^{18} \mathrm{~F}$-FDG PET/CT showed elevated metabolic activity of the RPLN, indicating that the SCC of the tongue had metastasized to the RPLN (fig. 1b). Considering various factors such as the patient's age and reoperation after neck dissection, the transoral approach was considered better than the transcervical approach because the dissection area affected by the transoral approach is smaller, and this approach allowed us to avoid any influence of scar tissue after neck dissection. In order to assess whether we could employ the transoral approach, we conducted contrast-enhanced CT with her mouth open, which showed that the enlarged RPLN clearly bound the surrounding tissue, including the carotid artery and the prevertebral muscles, and was localized below the level of the hard palate and the pterygoid hamulus. Sagittal-view contrast-enhanced CT demonstrated that the distances between the node and the surface of the pharyngeal wall and between the node and the submandibular skin were 16 and $72 \mathrm{~mm}$, respectively (fig. 1c, d). These findings indicated that the transoral approach was feasible and constituted minimally invasive surgery. Although CT and MRI findings suggested that the possibility of RPLN adhesion to the surrounding tissue was low, changing to the transcervical approach including 

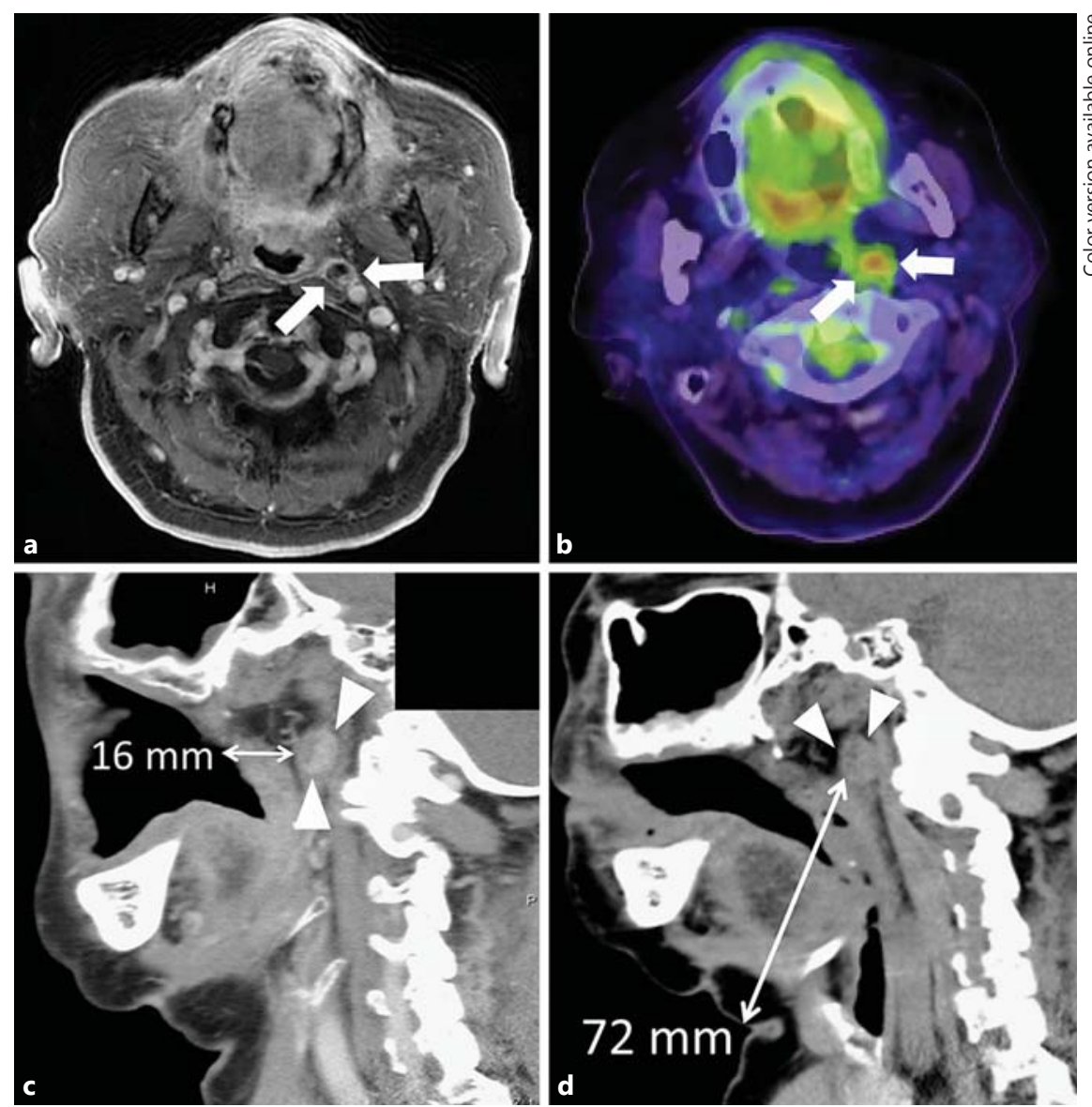

Fig. 1. a Gadolinium-enhanced T1-weighted MRI in an axial plane shows an enlarged left RPLN (arrows) nearly $2.0 \mathrm{~cm}$ in diameter with well-defined borders. b ${ }^{18} \mathrm{~F}$-FDG PET/CT shows elevated metabolic activity of the lymph node. c Sagittal-view contrast-enhanced CT with the mouth open demonstrated that the distance between the enlarged RPLN (arrowheads) and the surface of the pharyngeal wall was $16 \mathrm{~mm}$. $\mathbf{d}$ Sagittal-view CT with the mouth closed showed that the distance between the enlarged RPLN (arrowheads) and the surface of the submandibular skin was $72 \mathrm{~mm}$.

mandibulotomy was explained as part of an informed consent process in case of strong adhesion to the surrounding tissue.

TOVS with a navigation system was performed for an enlarged left RPLN. Under general anesthesia with oral endotracheal intubation, the patient was placed on the bed in the supine position with her head slightly extended from the neck. Figure 2 shows an overhead view of the operating room configuration for TOVS with a navigation system. The most appropriate surgical view was obtained using a Crowe-Davis retractor. A surgical assistant managed a high-definition videolaryngoscope to obtain the most appropriate view. We used a laryngeal rigid endoscope (12067VA; Karl Storz) with an Image 1 HD camera (Karl Storz). After exposing the surgical field, the left RPLN was not palpable, so the incision line could not be determined by endoscopic observation and palpation. By using the navigation system (Stealth Station TRIA plus, Medtronic, Switzerland), the location of the enlarged left RPLN was accurately identified and an incision line could be determined just above the RPLN. A curvilinear vertical incision was performed along the determined line medially to the left pterygomandibular raphe, using monopolar electrocautery (fig. 3a). The superior pharyngeal constrictor muscle was identified, exposing the underlying buccopharyngeal fascia. The fascia was incised, and the ascending pharyngeal artery was identified and clipped with hemoclips (8665 L/R; Karl Storz) (fig. 3b). Suction cautery (8606E; Karl Storz) was used for hemostasis. The capsule of the metastatic RPLN was identified and mobilized from the surrounding fibroadipose tissue (fig. 3c). The location of this 
Fig. 2. a Photograph during TOVS with a navigation system. $\mathrm{s}=$ Surgeon; $\mathrm{a}=$ surgical assistant; $\mathrm{ns}=$ navigation system; $\mathrm{r}=$ optical receiver of the navigation system; $\mathrm{nm}=$ navigation monitor; em = endoscopic monitor. b Overhead view of the operating room configuration for TOVS with a navigation system. An endoscopic monitor and a navigation monitor are placed on the opposite side of the surgical assistant to optimize hand-eye alignment of the surgeon during the surgery. The monitors are also situated such that the surgical assistant, the anesthesiologist, and nurses can confirm the surgical view and orientation.
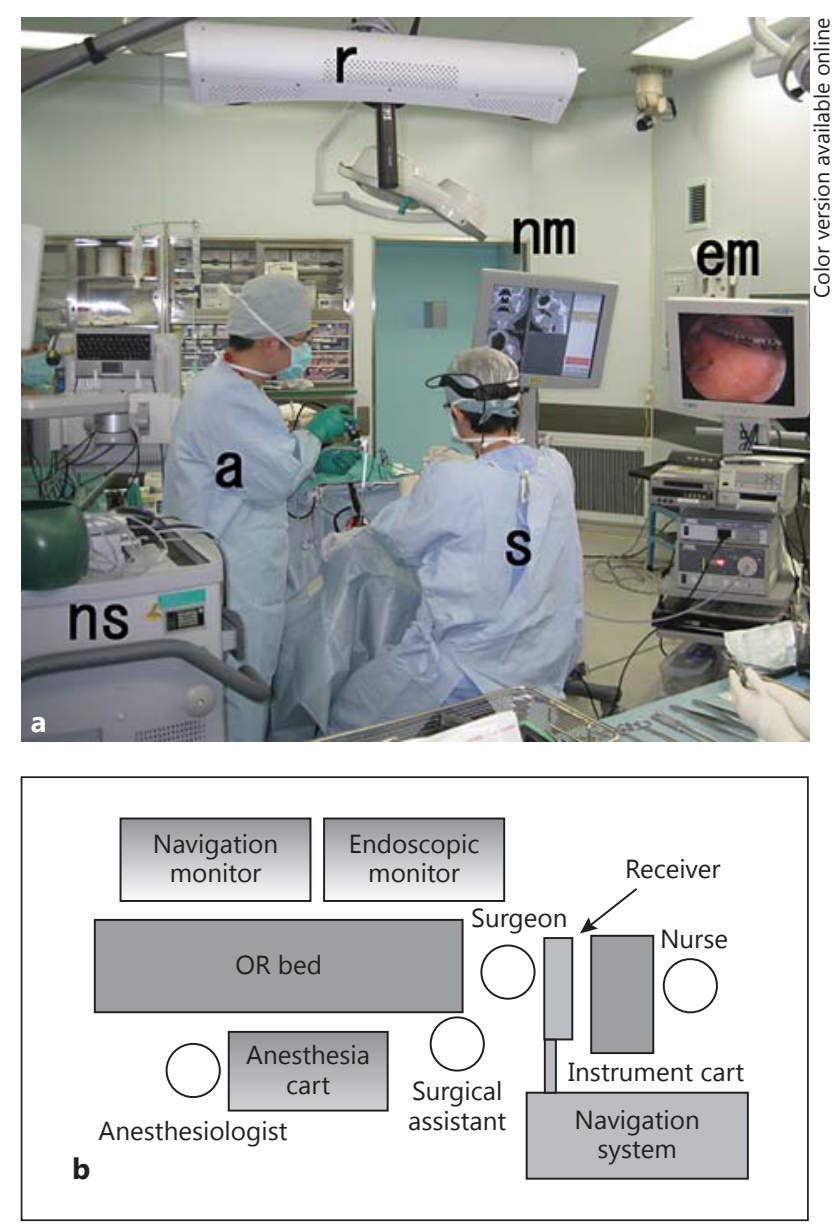

RPLN found by endoscopic observation and palpation coincided with that detected by the navigation system. The node was safely excised by blunt dissection. Care had to be taken to ensure that the node was not continuously joined with a nerve inferiorly, because the superior sympathetic ganglion may be mistaken for an RPLN. After the excision, the internal carotid artery was identified laterally, and the alar fascia posteriorly (fig. 3d). After meticulous hemostasis, the incision was closed in layers with interrupted absorbable sutures.

Videofluorography performed on the first postoperative day confirmed that the swallowing function was not impeded. Oral feeding was started on the first postoperative day. Horner's sign and cranial nerve damage were not evident, and the postoperative course was uneventful. Pathological analysis showed a metastatic SCC.

Postoperative radiation therapy (60 Gy in 30 fractions) was conducted as a preventive measure. No recurrence was apparent more than 1 year after the surgery.

\section{Discussion}

Various surgical approaches to the RPS have been reported for the treatment of RPS lesions, since the RPS is difficult to access. The key to successfully operate RPS lesions is to ensure an adequate surgical field as well as an adequate view enabling the identification of surrounding vital nerves and vessels. Surgical approaches to the RPS are classified as transcervical [5] or transoral [9-11]. A transcervical approach is conventionally chosen as an extension of neck dissection. However, it entails various complications, such as damage to the critical nerves and vessels due to the limited surgical field, dysphasia, and aspiration neces- 

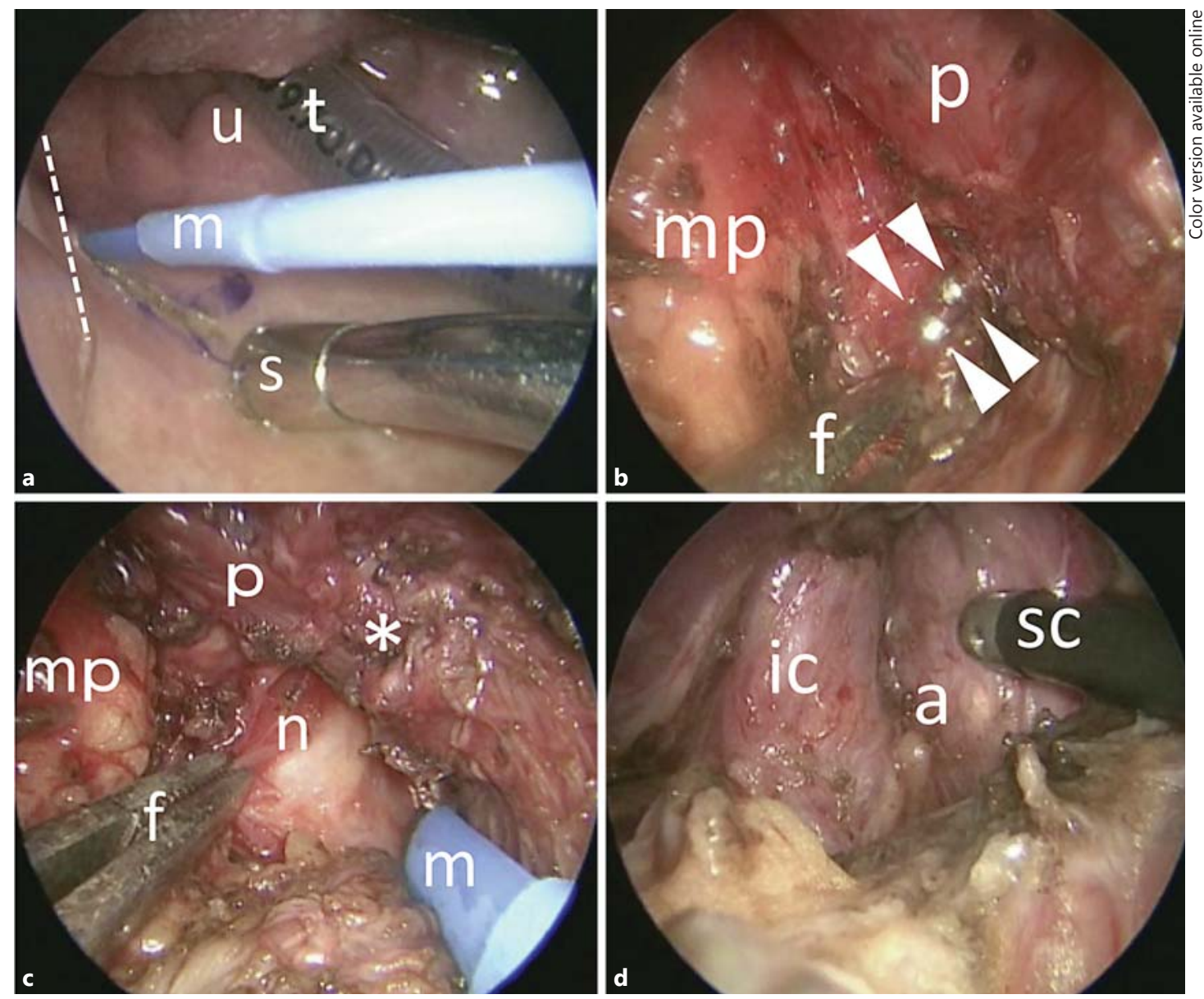

Fig. 3. Intraoperative endoscopic view of TOVS. a Incision of the mucosa was performed along the determined line medial to the left pterygomandibular raphe (white broken line) using monopolar electrocautery $(\mathrm{m}) . \mathrm{s}=$ Suction; $\mathrm{t}=$ endotracheal tube; $\mathrm{u}=\mathrm{uvula}$. $\mathbf{b}$ The ascending pharyngeal artery (arrowheads) was identified and clipped. $\mathrm{mp}=$ Medial pterygoid muscle; $\mathrm{p}=$ superior pharyngeal constrictor muscle; $\mathrm{f}=$ forceps. $\mathbf{c}$ The buccopharyngeal fascia (asterisk) was incised, and the fascia of the enlarged lymph node (n) was identified. $\mathbf{d}$ After the excision, the internal carotid artery (ic) was identified and preserved. a = Alar fascia; $\mathrm{sc}=$ suction coagulator.

sitating a gastrostomy tube and tracheotomy, especially in cases involving mandibulotomy [12].

The transoral approach has recently been reported as a minimally invasive and easy access route for the removal of RPS tumors. The conventional transoral approach has certain disadvantages, such as an inadequate surgical field and view as well as disturbance of the surgeon's depth perception. Shellenberger et al. [10] reported 3 cases of differentiated thyroid carcinoma safely removed by a transoral approach guided by preoperative ultrasonography. Byeon et al. [11] also reported the removal of parapharyngeal tumors by transoral robotic surgery. Liu et al. [13] noted the usability of intraoperative cone beam CT guidance with transoral robotic surgery in the oropharyngeal region of porcine and cadaveric models. By using assistive modalities including ultrasonography, cone beam CT, and a robotic system, the transoral approach is applicable to not only benign RPS tumors but also malignancies. In addition, it was necessary to identify accurate anatomical structures surrounding RPLNs to avoid injury to vital nerves and vessels; therefore, we considered applying a navigation system to 
the transoral approach to RPS. To our knowledge, this is the first case in which the transoral approach with a navigation system has been applied to a metastatic RPLN of SCC.

Optical navigation systems have been known as being useful for ensuring precise surgical orientation and, furthermore, to educate less experienced surgeons in head and neck regions including the paranasal sinus and the skull base region. However, there are few reports of navigation systems being applied to the laryngopharyngeal region, since neck extension and mouth opening during the surgery cause a gap between real-time and preacquired images. The RPS, however, is an appropriate area for the utilization of a navigation system in the laryngopharyngeal region, as the RPS is less susceptible to mobility caused by neck extension than the other laryngopharyngeal regions. In the case reported herein, misregistration did not occur during surgery, and it was very useful to determine the incision line just above the RPLN and to identify the RPLN in the RPS. A potential problem with the application of an optical navigation system to RPS surgery is that the operating room configuration can cause disturbance of optical tracking if the surgeon's body is located between the optical sensor and the receiver. To solve this problem, an electromagnetic navigation system might be useful, since the sensor and receiver can be set around the patient's head, excluding the influence of the surgeon's position.

Our experiences with metastatic RPLNs suggest that indications for the transoral approach to the RPS include (1) that the location of the RPLN is below the level of the hard palate and the pterygoid hamulus when the mouth is open and (2) that there is only a single lesion present, without adhesion to the surrounding tissue. The first indication is based on the fact that the transoral approach is limited by the degree of mouth opening, and we consider the level of the hard palate and the pterygoid hamulus to accurately predict the cephalic limit of surgical exposure of the RPS. To verify the clinical value of CT when the mouth is open, however, more data need to be accumulated. The second indication is based on the fact that transoral surgical exposure of the RPS is limited. In our opinion, cases with multiple metastases in the RPS or extracapsular spread of metastatic RPLNs should be excluded with respect to curability and avoidance of damage to vital nerves and vessels. Thin-slice CT and MRI are useful for evaluating adhesion of RPLNs to the surrounding tissue; however, the possibility of changing to the transcervical approach including mandibulotomy due to unexpected adhesion or uncontrollable bleeding should be explained preoperatively.

\section{Conclusions}

TOVS with a navigation system for excision of a metastatic RPLN was safely performed. This is the first case in which a transoral approach with a navigation system was applied to a metastatic RPLN of SCC. Indications for the transoral approach in cases of such malignancies are that the RPLN is not adherent to the surrounding tissue and that it is localized below the level of the hard palate and the pterygoid hamulus when the mouth is open. TOVS with a navigation system can be performed as a safe and minimally invasive surgical procedure for metastasis to the RPLN.

\section{Acknowledgements}

This research was partly supported by grants-in-aid for Scientific Research (C; KAKENHI 25462699) from the Japan Society for the Promotion of Science and a Health and Labor Sciences Research Grant for Clinical Cancer Research (H21-Gannrinshou-Ippan-016) from the Ministry of Health, Labor and Welfare, Japan. 
Tanaka et al.: Transoral Videolaryngoscopic Surgery with a Navigation System for Excision of a Metastatic Retropharyngeal Lymph Node

\section{References}

1 Ballantyne AJ: Significance of retropharyngeal nodes in cancer of the head and neck. Am J Surg 1964;108: 500-504.

-2 Hasegawa Y, Matsuura H: Retropharyngeal node dissection in cancer of the oropharynx and hypopharynx. Head Neck 1994;16:173-180.

-3 Morrissey DD, Talbot JM, Cohen JI, Wax MK, Andersen PE: Accuracy of computed tomography in determining the presence or absence of metastatic retropharyngeal adenopathy. Arch Otolaryngol Head Neck Surg 2000; 126:1478-1481.

-4 Amatsu M, Mohri M, Kinishi M: Significance of retropharyngeal node dissection at radical surgery for carcinoma of the hypopharynx and cervical esophagus. Laryngoscope 2001;111:1099-1103.

-5 Ozlugedik S, Ibrahim Acar H, Apaydin N, Firat Esmer A, Tekdemir I, Elhan A, Ozcan M: Retropharyngeal space and lymph nodes: an anatomical guide for surgical dissection. Acta Otolaryngol 2005;125:1111-1115.

6 Shiotani A, Tomifuji M, Araki K, Yamashita T, Saito K: Videolaryngoscopic transoral en bloc resection of supraglottic and hypopharyngeal cancers using laparoscopic surgical instruments. Ann Otol Rhinol Laryngol 2010; 119:225-232.

-7 Shiotani A, Tomifuji M, Araki K, Yamashita T: Transoral videolaryngoscopic surgery for en bloc resection of supraglottic and hypopharyngeal cancers. Otolaryngol Head Neck Surg 2011;144:288-289.

-8 Yamashita T, Tomifuji M, Araki K, Kurioka T, Shiotani A: Endoscopic transoral oropharyngectomy using laparoscopic surgical instruments. Head Neck 2011;33:1315-1321.

-9 Le TD, Cohen JI: Transoral approach to removal of the retropharyngeal lymph nodes in well-differentiated thyroid cancer. Laryngoscope 2007;117:1155-1158.

10 Shellenberger T, Fornage B, Ginsberg L, Clayman GL: Transoral resection of thyroid cancer metastasis to lateral retropharyngeal nodes. Head Neck 2007;29:258-266.

11 Byeon HK, Duvvuri U, Kim WS, Park YM, Hong HJ, Koh YW, Choi EC: Transoral robotic retropharyngeal lymph node dissection with or without lateral oropharyngectomy. J Craniofac Surg 2013;24:1156-1161.

12 Olsen KD: Tumors and surgery of the parapharyngeal space. Laryngoscope 1994;104(5 pt 2 suppl 63):1-28.

13 Liu WP, Reaugamornrat S, Sorger JM, Siewerdsen JH, Taylor RH, Richmon JD: Intraoperative image-guided transoral robotic surgery: pre-clinical studies. Int J Med Robot 2014, Epub ahead of print. 